First Peoples Child \& Family Review

An Interdisciplinary Journal Honouring the Voices, Perspectives, and Knowledges of

First Peoples through Research, Critical Analyses, Stories, Standpoints and Media

Reviews

\title{
Non-Aboriginal Child Protective Service Workers' Utilization of the Maltreatment and Adolescent Pathway Knowledge Translation (MAP-KT) Portal: A Report on the Utilization Statistics and Utilization Gaps of Aboriginal Best-Practice Material
}

\author{
Eman Leung, Christine Wekerle, Randy Weachter, Julian Egelstaff and Marlyn \\ Bennett
}

Volume 6, Number 2, 2011

URI: https://id.erudit.org/iderudit/1068872ar

DOI: https://doi.org/10.7202/1068872ar

See table of contents

Publisher(s)

First Nations Child and Family Caring Society of Canada

ISSN

1708-489X (print)

2293-6610 (digital)

Explore this journal

Cite this article

Leung, E., Wekerle, C., Weachter, R., Egelstaff, J. \& Bennett, M. (2011).

Non-Aboriginal Child Protective Service Workers' Utilization of the

Maltreatment and Adolescent Pathway Knowledge Translation (MAP-KT)

Portal: A Report on the Utilization Statistics and Utilization Gaps of Aboriginal

Best-Practice Material. First Peoples Child \& Family Review, 6(2), 7-13.

https://doi.org/10.7202/1068872ar

\section{Article abstract}

Given the unique history and culture of the Aboriginal heritage, research evidence and evidence-based practice guidelines/recommendation derive from the general population are not sufficient in informing the practices of Child Protective Services' caseworkers who are caring for Aboriginal youth in the child welfare system. Special attention should also be given to best-practice materials developed with special consideration of the Aboriginal context. The current study describes caseworkers' utilization of Aboriginal child welfare best-practice materials hosted at the MAP-KT portal during its pilot testing period between October 1st 2007 and September 31st 2008. The objective of the current study is to assess the knowledge consumption behavior of caseworkers who were not employed by Aboriginal child protective agency but nonetheless had Aboriginal Child Welfare youth under they care. The MAP-KT portal is a web-based knowledge tool that brings to the point of practice (of CPS caseworker) child welfare knowledge distilled through the "knowledge filter" of the Knowledge to Action framework. Given the proportion of Aboriginal youth under the care of participating agencies, the utilization statistics of Aboriginal content hosted on the MAP-KT portal indicated that they might be under-utilized compared to other content areas. According to the Knowledge to Action framework, a number of ways to improve utilization have been proposed. Future research may focus on adapting the MAP-KT portal to user knowledge preferences on format, content and linkage to frequently utilized sites, as well as tailored marketing to users in First Nations child welfare services and those who provide services to children with First Peoples' heritage.
This document is protected by copyright law. Use of the services of Erudit (including reproduction) is subject to its terms and conditions, which can be viewed online. 


\section{First Peoples Child \& Family Review}

An Interdisciplinary Journal Honoring the Voices, Perspectives and Knowledges of First Peoples through Research, Critical Analyses, Stories, Standpoints and Media Reviews

\section{Non-Aboriginal Child Protective Service Workers' Utilization of the Maltreatment and Adolescent Pathway Knowledge Translation (MAP-KT) Portal: A Report on the Utilization Statistics and Utilization Gaps of Aboriginal Best-Practice Material}

\author{
Eman Leung ${ }^{a}$, Christine Wekerle ${ }^{\mathrm{b}, \mathrm{c}, \mathrm{d},}$ Randy \\ Weachter ${ }^{\mathrm{b}}$, Julian Egelstaffe, and Marlyn \\ Bennett ${ }^{f}$ \\ a Knowledge Translation Program, Li Ka Shing Knowledge Institute, St. \\ Michael's Hospital, Toronto, ON, Canada \\ ${ }^{b}$ McMaster University, ON, Canada \\ c The University of Western Ontario, ON, Canada \\ d Canadian Institutes of Health Research Centre, Prevention of Violence \\ Across the Lifespan, Toronto, ON, Canada \\ e Freeform Solutions, Toronto, ON, Canada \\ ${ }^{f}$ First Nations Child \& Family Caring Society of Canada, Manitoba Site, \\ Canada
}

\section{Introduction}

Aboriginal children are over-represented in child protective services (CPS; Auditor General of Canada Report, 2008, http:// www.oag-bvg.gc.ca/internet/English/parl_ oag_200805_04_3_30700.html; foradiscussion, see Blackstock, 2009, http://www.fncfcs.com/ docs/ChildWelfareTribunalBriefingNote.pdf). Based on analysis of a number of CPS data sources wherein non-Aboriginal agencies were over-represented, an Ontario report (Wekerle, Waechter, Leung, \& Chen, 2009) noted that,

Questions or correspondence concerning this article may be addressed directly to:

Christine Wekerle

Pediatrics

McMaster University

1200 Main St. W., Hamilton, ON, L8N $3 Z 5$

Email: chris.wekerc@mcmaster.ca

\begin{abstract}
Given the unique history and culture of the Aboriginal heritage, research evidence and evidence-based practice guidelines/ recommendation derive from the general population are not sufficient in informing the practices of Child Protective Services' caseworkers who are caring for Aboriginal youth in the child welfare system. Special attention should also be given to best-practice materials developed with special consideration of the Aboriginal context. The current study describes caseworkers' utilization of Aboriginal child welfare best-practice materials hosted at the MAP-KT portal during its pilot testing period between October 1st 2007 and September 31st 2008. The objective of the current study is to assess the knowledge consumption behavior of caseworkers who were not employed by Aboriginal child protective agency but nonetheless had Aboriginal Child Welfare youth under they care. The MAP-KT portal is a web-based knowledge tool that brings to the point of practice (of CPS caseworker) child welfare knowledge distilled through the "knowledge filter" of the Knowledge to Action framework.
\end{abstract}

Given the proportion of Aboriginal youth under the care of participating agencies, the utilization statistics of Aboriginal content hosted on the MAP-KT portal indicated that they might be under-utilized compared to other content areas.

According to the Knowledge to Action framework, a number of ways to improve utilization have been proposed. Future research may focus on adapting the MAP-KT portal to user knowledge preferences on format, content and linkage to frequently utilized sites, as well as tailored marketing to users in First Nations child welfare services and those who provide services to children with First Peoples' heritage.

Keywords: Knowledge Translation, Aboriginal, Child Protective Services, Child Welfare, Child Abuse and Neglect, Health Outcomes

in 2006 , about $16 \%$ of out-of-home care youth sampled from mostly non-Aboriginal agencies in Ontario were Aboriginal children, and most of these youths were noted to be involved in some form of Aboriginal cultural practices. 


\section{Acknowledgements:}

We thank the Centre of Excellence in Child and Youth Mental Health at the Children's Hospital of Eastern Ontario for funding the development and preliminary evaluation of the MAP-KT site. Also, we thank the Ontario Association of Children's Aid Societies for their support of the MAP-KT site, and child welfare advisors to the MAP-KT, including Brenda Moody, Bruce Leslie, and Deborah Goodman. Many persons provided input to the MAP-KT and we especially acknowledge Lisa van Krieken, Maria Chen, Jennifer Fong, Andrew Fong, and Freeform Solutions for their efforts. Dr. Wekerle acknowledges career funding from Canadian Institutes of Health Research, Institute of Gender and Health/ Ontario Women's Health Foundation, and a Canada Interchange Agreement with the Public Health Agency of Canada.

Because of the impact of residential school experience on family functioning and the multigenerational losses experienced among First Nations people, Mussell, Cardiff and White (2004) suggested that an opportunity for actions could be created only when the cultural determinants of health and well-being of Aboriginal children are recognized. While evidence-based child welfare practices appropriate to the Aboriginal context is still developing, fostered in large part by scholarly journals focused on Aboriginal research and issues, there is already a substantial body of research that may be useful to agencies and caseworkers who provide services to First Peoples' children and youth. This creates a need and an opportunity to translate knowledge from Aboriginal child welfare research into evidencebased practice for caseworkers caring for First Peoples' children and youth in Aboriginal and non-Aboriginal agencies.

According to the Canadian Institutes of Health Research (CIHR), knowledge translation (KT) is "the exchange, synthesis and ethically-sound application of knowledge - within a complex system of interactions among researchers and users - to accelerate the capture of the benefits of research for Canadians through improved health, more effective services and products, and a strengthened health care system" (http://www. cihr.ca/e/26574.html). Hence, for knowledge translation to achieve its intended impact in the area of Aboriginal child welfare, it is critical for researchers and Aboriginal child welfare practitioners to engage in knowledge exchange, to synthesize research knowledge into a format accessible to end-users, and to apply them in an ethical, effective and sustainable manner. To this end, we have developed the MAP-KT portal according to the Knowledge To Action Framework to facilitate the exchange, synthesis and application of knowledge within the context of child welfare practices. The MAP-KT portal is a web-based knowledge tool that brings knowledge distilled through the "knowledge filter" (Graham et al, 2006) to caseworkers at the point of practice.

\section{The Aboriginal Research Context in Canada: Use of Published Knowledge}

$\mathrm{KT}$ has emerged as a unique area wherein knowledge is implemented and its implementation evaluated (Schryer-Roy, 2005). According to the Knowledge To Action Framework (Graham et al, 2006), the process that moves knowledge to action begins with the process of distilling unrefined scientific studies into knowledge tools, which are then adapted to local context of practice and implemented after identifying and overcoming barriers for knowledge use; finally, the implementation of knowledge is evaluated, monitored and sustained. Within the context of the Aboriginal knowledge tradition, knowledge is already inherently practical (Estey, Smylie \& Macaulay, 2009). The authors reminded researchers and policy-makers interested in Aboriginal health to learn from and integrate into their work the unique ethical dimension as well as the different understanding of 'knowledge' and 'translation' specific to the Aboriginal community. CIHR has put into effect guidelines focusing on demonstrable relationship development and engagement that are respectful of cultural practices and values, to which research on First Peoples' populations should adhere (http://www. cihr-irsc.gc.ca/e/29339.html). The guidelines highlight the 4 R's of research - respect, reciprocity, relevance, and responsibility, and they provide a framework for understanding 
and engaging in Aboriginal ethics. Estey, Smylie and Macaulay (2009) also suggest that many sources of knowing is necessary to fill the large gaps in our knowledge concerning Aboriginal health issues, and thus different types and sources of knowledge must be understood and respected by the world of research and policy. Finally, the authors caution practitioners of KT that the translation of knowledge into action within the Aboriginal context necessarily requires community input and support at the onset, which entails the involvement of Aboriginal Peoples in all research and implementation activities (see CIHR's definition of Integrated KT: http:// www.cihr.ca/e/39033.html). Hence, due to the unique culture and history of the First Peoples, evidence-based practices in Aboriginal child welfare are best informed by knowledge specific to the Aboriginal context in addition to knowledge developed from the general population. The current study describes the utilization statistics of Aboriginal child welfare research evidence and evidence-based guidelines / recommendations among caseworkers who cared for Aboriginal child welfare youth but were not employed under the First Nations Child Welfare Authorities.

To disseminate child welfare research evidence to the caseworkers of Ontario Aboriginal and non-Aboriginal youth, evidence-based guidelines and recommendations of child welfare practices are hosted on the Maltreatment and Adolescent Pathways Project Knowledge Translation (MAPKT) portal for open access. The MAP-KT portal was designed to provide the broader Ontario child welfare community with a wide access to research on adolescent development and special interest areas, and a quick access to outcome statistics and evidence-based practice guideline/ recommendation across areas such as mental health, substance use, dating violence and risky sexual behaviors.

Here, we report the utilization of a KT tool and the gap its utilization statistics reveals. We recognize the existence of several child welfare resources and platforms for exchanging knowledge, and the MAP-KT portal is only one example within this category. And while many means of disseminating knowledge are available, we decided to provide relevant best- available evidence through the Internet to facilitate easy access to high quality information, furnishing opportunities for communication with tool developers to support and sustain evidencebased practice (for a systematic review on the validity and efficacy of online knowledge tools in one health area, see Yu, Bhaniwal, Laupacis, Leung, Orr and Straus, in press).

\section{The Maltreatment and Adolescent Pathways-Knowledge Translation (MAP-KT) Portal}

As a KT spin-off of the Maltreatment Adolescent Pathways (MAP) project (Waechter et al, 2009; Wekerle, Leung, MacMillan, Boyle, Trocmé, \& Waechter 2009; Waechter, Leung, Wekerle et al., 2011), the MAP-KT portal was developed with the primary objective of bringing relevant research evidence to Child Welfare agency employees, policymakers, and partners in a facilitated way in order to inform decision-making. The secondary objective was to maintain an open access to research and provide tangible opportunities for partnership between frontline practitioners and university academics. The MAP-KT is, therefore, available to all the members $(50,000+)$ of the Ontario Association of Children's Aid Societies (OACAS) website, which is the prime communication vehicle for practice and policy for the 53 CPS agencies in the province of Ontario.

In alignment with the two objective stated above, the MAP-KT is consisted of five sections: 1) a running web-log where practice-relevant evidence is reviewed in brief by content experts in the area; 2) a scholarly article search function that is designed to source high impact and highly relevant articles, with searches pre-restricted to child welfare-relevant areas; 3) a best-practice search function that provides portable digital files of evidence-based clinical guidelines, screenings tools and recommendations on child welfare practices pre-vetted by the investigators of the MAP study according to the latest evidence in child welfare research; 4) an automatized outcomes statistics section that compares child welfare youth to population-based samples of youth across a number of areas [based on the 
epidemiological data collected from the MAP project and other published statistics from national databases such as the OSDUHS (Adlaf and Paglia-Boak, 2007)]; 5) a researcher-oncall service, providing OACAS members with a personalized response to their specific child welfare inquiries that are evidence-based and expert-driven. As the research emerges in the field along specified priority content areas, the MAP-KT portal is updated, providing the opportunity for child welfare practitioners to access research trends. Users' knowledge consumption behavior is tracked automatically on the MAP-KT portal.

In the current study, we report the MAPKT portal utilization statistics with respect to access of Aboriginal materials, and the nature and frequency of search and query in relation to Aboriginal-related keywords or topics. Only utilization statistics from non-Aboriginal CPS agencies will be reported. Since the end-users of the MAP-KT portal came from the same population of caseworkers who participated in the MAP project (no Aboriginal agency was participated), and since $10 \%$ of all youth in the MAP project reported an Aboriginal heritage (Waechter, Leung, Wekerle et al., 2011), it is expected that at least $10 \%$ of all traffic captured by the MAP-KT portal should be accessing Aboriginal materials. A key advantage of accessing the MAP-KT portal is that the posted research information is pre-vetted to include only evidence that have already gone through the peer-reviewed processes to ensure a level of critical review that conforms to the current scholarship standards. While searches for scholarly work on sites such as Google Scholar prioritize academic articles according to their citation indices, often these are only available commercially. Also, while the search for content via general search engines may link to useful information, it is not peer-reviewed, and as such the quality and accuracy of the information are unknown and the information presented may be substantially biased (with biases undeclared). The goal of the MAP-KT portal is to adhere to a high scientific standard in the quality of information provide while addressing the practical needs through its method of delivery, such as blogs, search engine of themespecific research evidence and evidence-based practice guidelines/recommendations, and the researcher-on-call service.

\section{Method}

The MAP-KT usage had ethics approval from the University of Western Ontario and received input from the child welfare representatives on the MAP Advisory Board. User data during this pilot period (Oct 1st 2007 to Sept 31st 2008) was used to examine the usage of the MAP-KT portal, and the nature of the content searched or requested. When members logged onto the site with their agency-specific e-mail address, the usage of each service by each account was tracked. All member agencies of the OACAS received e-alerts about the MAP-KT portal resource. Access to the MAP-KT portal was hosted on the homepage of the OACAS member website, and was a visible presence with each homepage loading. Finally, banners about the MAP-KT website ran across the OACAS homepage consistently during the pilot testing period.

The MAP-KT portal, being in a developmental phase, was not actively promoted (with the exception of agencies involved with the MAP project), so as to capture a natural flow of site traffic and the level of usage of available resources. It was introduced to an Ontario network of child welfare quality assurance, actively marketed to the child welfare agencies involved in the MAP project and presented at the First Nations Child and Family Caring Societies (FNC\&FCS) conference "Bridging the Gap" (http://www.kidsmentalhealth.ca/documents/ Res_MP14.pdf).

Due to ethical and privacy concerns, we did not capture any demographic information or professional profile from caseworkers who accessed the MAP-KT portal. Only utilization statistics from non-Aboriginal CPS agencies is reported below. Since the end-users of the MAP-KT portal came from the same population of caseworkers who participated in the MAP project (no Aboriginal agency has participated), 
and since $10 \%$ of all youth in the MAP project reported an Aboriginal heritage (Waechter, Leung, Wekerle et al., 2011), it is expected that at least $10 \%$ of all traffic captured by the MAP-KT portal should be accessing Aboriginal materials

\section{Results}

Between the period of October 1st 2007 and Sept 31st 2008, usage statistics of each section in the MAP-KT portal was as follows: the homepage that contains RSS feeds of child welfare research news and high interest materials, including the banner of the FNC\&FCS and articles from the FNC\&FCS, was visited 3,045 times. Theme-specific annotated journal articles posted on the homepage were accessed 928 times. Best-practice materials (including pre-vetted clinical guidelines, screenings tools and recommendations on child welfare practices across areas such as mental health, substance abuse, Aboriginal child welfare) were accessed 1,400 times. Outcome-statistics (based on the epidemiological data collected from the MAP project and other published statistics from national databases such as the OSDUHS) was accessed 1,157 times. Finally, the researcheron-call service, which allowed for a specific matching between an inquiry about a program, policy area, or clinical issue, with samples from the research literature, was used 58 times.

Of the 3,045 visits to the homepage, 56 visits (2\%) involved clicking on the banner of the FNC\&FCS website and articles from the FNC\&FCS. In terms of theme-based search for best-practice material, less than $1 \%$ of all who conducted theme-based search on child welfare best-practice guidelines requested materials pertaining to the Aboriginal populations. In terms of specific keywords used in the Google Scholar search engine (pre-set by the MAP-KT team to select child welfare or maltreatment publications): $10 \%$ was associated with pregnancy and parenting, $7 \%$ with education, $6 \%$ with homelessness, $3 \%$ with sexual orientation, and $2 \%$ with youth justice, with only less than $1 \%$ of searches contained "Aboriginal" or "First Nations" as keywords. In terms of the personalized query where users submitted directly to the researcher-on-call tool,
$28 \%$ of all queries submitted in this interactive format were associated with behavioral outcomes and mental health, $48 \%$ with child welfare-specific activities. Again, the content specific areas for the researcher-on-call services were: $12 \%$ were associated with parenting, $5 \%$ with education, $5 \%$ with youth justice, and only $2 \%$ were associated with Aboriginal/First Nations issues.

\section{Discussion}

Given the over-representation of Aboriginal children in child welfare, and the finding that $10 \%$ of youth randomly sampled from non-Aboriginal child welfare agencies reported having at least 1 Aboriginal birth parent (Waechter, Leung, Wekerle et al., 2011), caseworkers may have under-utilized Aboriginal content on the MAPKT portal as compared to other contents. As a result, the MAP-KT portal was under-utilized as a potential tool to bridge between knowledge and action in the practice of caring for Aboriginal children and youths in the child welfare system.

According to the Knowledge-to-Action framework (Graham et al, 2006), a knowledge tool (such as the MAP-KT portal) is the end point of a distillation process where unrefined knowledge is turned into usable form. According to Graham et al. (2006), the knowledge-to-action cycle requires: 1) adapting the tool to local practice context, 2) identifying barriers to knowledge use, and 3) selecting, tailoring and implementing intervention according to the barriers identified. Hence, the utilization of the MAP-KT portal in Aboriginal child welfare practices could be improved by reviewing the format and selection of information on the MAP-KT portal, and ensuring that the information is adapted to the needs and preferences of end-users who are responsible for the well-being of Aboriginal children involved in CPS.. Harrison, Graham, and Fervers (2009) suggest that customizing a knowledge tool for a particular organization or culture may help improve the acceptance of and adherence to tool use. A customized knowledge tool also helps to overcome challenges such as the lack of expertise among service providers in implementing recommended actions, the lack of 
mandatory equipment or staff time to deliver a guideline's recommendations, or the rejection of recommendations by the local patient population or by the providers due to culture or other factors (Harrison, Graham, \& Fervers, 2009). The authors recommend using the ADAPTE process (The AGREE Collaboration, 2004; see also Fervers et al, 2006) to adapt a knowledge tool to its local context. The ADAPTE process was developed to facilitate the creation of high-quality knowledge tools with a higher likelihood of being successfully implemented. The process engages end-users in the adaptation of the knowledge tool to address specific questions relevant to its use. The goal is to establish a transparent, rigorous, and replicable standard based on the following core principles: 1) respect for evidencebased principles in knowledge tool development; 2) use of reliable and consistent methods to ensure the quality of the adapted knowledge tool; 3) participation of key stakeholders to foster acceptance and ownership of the adapted knowledge tool, and ultimately to promote its use; 4) consideration of context during adaptation to ensure relevance for local practice and policy; 5) transparent reporting to promote confidence in the recommendations of the adapted guideline; 6 ) use of a flexible format to accommodate specific needs and circumstances; and 7) respect for and acknowledgment of source guideline materials (The AGREE Collaboration, 2004). Such adaptation process could be the next phase of development for the MAP-KT portal.

The activity to overcome barriers to the successful implementation of a knowledge tool is called knowledge translation intervention (Wensing, Bosch, \& Grol, 2009). According to the authors, knowledge translation interventions need to be tailored to specific barriers for change, similar to a clinical treatment tailored to a diagnosed health problem. One of the most often cited conceptual frameworks regarding barriers to knowledge use in health care is the Clinical Practice Guidelines Framework for Improvement (Cabana et al, 1999). This framework was based on an extensive search of the literature of barriers to physician adherence to clinical practice guidelines (an example of a knowledge tool). Three main categories of barriers were identified: knowledge, attitudes, or behavior of the practitioners. Based on a systematic approach to evidence, knowledge tools such as clinical practice guidelines, are defined as systematically developed statements to assist practitioners and clients in making decisions about appropriate health and social services under specific circumstances (for a detailed description of different board categories of barriers to knowledge tool implementation and their corresponding interventions, refer to Wensing, Bosch, \& Grol, 2009). To clearly identify barriers and facilitators to knowledge use in health care and social service practice, there is a need to assess them in a valid and reliable fashion. In this context, considerable interest is generated in instruments for valid and reliable assessment of barriers and facilitators to knowledge use that can be employed by various end-users who are trying to implement knowledge (Légaré, 2009).

The current study demonstrates that the caseworkers may have under-utilized Aboriginal content as compared to other contents hosted on the MAP-KT portal, suggesting that there is a gap in using the MAP-KT portal to bridge between knowledge and action in the practice of caring for Aboriginal children and youths in the child welfare system. Recommendations to increase the utilization of MAP-KT portal in the context of Aboriginal care include systematic adaptation of the knowledge tool to the local context, comprehensive assessment of barriers to tool use, and tailored intervention to promote tool use according to the barriers identified.

It is important to note that the results reported here are based on data collected within a limited time frame (under one year), during which no formal marketing of the MAP-KT site occurred. Thus, this report captures the "natural" information-seeking activities of OACAS members across the province of Ontario and is best viewed within a pilot-study context. With increasing scholarship in the area of Aboriginal child welfare, further research into the broader usage of such knowledge is warranted. It is clear that evidence-based or evidence-informed practice needs to build on a good fit between the 
knowledge users and the evidence. The ultimate aim is to ensure that the well-being of Aboriginal children is fully supported by the best available knowledge. Child welfare practitioners require the time and impetus to identify the most useful scholarship, and to feed back to the research field the practice-relevant questions that need to be answered. More knowledge tends to lead to more questions, questions that are increasingly more tailored to the population being served.

\section{Reference}

Auditor General of Canada Report (2008). First Nations Child and Family Services Program_Indian and Northern Affairs Canada. Retrieved from http://www.oag-bvg. gc.ca/internet/English/parl_oag_200805_04_e_30700. html (accessed June, 2010).

Blackstock, C. (2009). Child Welfare Tribunal Briefing Note: First Nations Child and Family Caring Society and the Assembly of First Nations VS. The Attorney General of Canada. Retrieved from http://www.fncfcs.com/docs/ ChildWelfareTribunalBriefingNote.pdf (accessed June, 2010)

Cabana MD, Rand CS, Powe NR, Wu AW, Wilson MH, Abboud PA, \& Rubin HR (1999). Why don't physicians follow clinical practice guidelines? A framework for improvement. JAMA, 282(15), 1458-65.

Estey, E., Smylie, J., \& Macaulay, A. (2009). Aboriginal Knowledge Translation Understanding and Respecting the Distinct Needs of Aboriginal Communities in Research. Retrieved from http:www.cihr-irsc. gc.ca/e/41392.html (accessed June, 2010).

Fervers B, Burgers JS, Haugh M, Latreille J, Mlika-Cabanne N, Paquet L, et al. (2006). Adaptation of clinical guidelines: a review of methods and experiences. Int $J$ Health Care, 18(30), 167-76.

Graham, I. D., Logan, J., Harrison, M. B., Straus, S. E., Tetroe, J., Caswell, W., \& Robinson, N. (2006). Lost in knowledge translation: Time for a map? Journal of Continuing Education in the Health Professions, 26(1), 13-24.

Harrison, MB., Graham, ID., and Fervers, B. (2009), Adapting knowledge to a local context. In S. Straus, J. Tetroe, and I. Graham (Eds.) Knowledge Translation in Health Care: Moving from Evidence to Practice (pp.7382). Wiley: UK
Légaré, F. (2009). Assessing barriers and facilitators to knowledge use. In S. Straus, J. Tetroe, and I. Graham (Eds.) Knowledge Translation in Health Care: Moving from Evidence to Practice (pp.83-93). Wiley: UK

Mussell, B., Cardiff, K., \& While, J. (2004). Mental health and well-being of Aboriginal children and youth: Annotated bibliography. A Report Prepared for the British Columbia Ministry of Children and Family Development.

Schryer-Roy, A. (2005). Knowledge translation: Basic theories, approaches and applications. Ottawa, ON: IDRC Research Matters in Governance, Equity and Health.

The AGREE Collaboration. (2004). International assessment of quality clinical practice guidelines in oncology using the Appraisal of Guidelines and Research and Evaluation Instrument. J Clin Oncol, 22, 2000-2007.

Waechter, R., Wekerle, C., Leslie, B., Goodman, D., Wathen, N., Moody, B., Bennet, M. \& The MAP Research Team (Boyle, M., Leung, E., MacMillan, HL., Trocmé, N.) (2009). Child Protection Services and university-based partnerships: A Participatory Action-Based Model for creating and sharing knowledge. First Peoples Child and Family Review, 4(2), 118-128.

Waechter, R., Leung, E., Wekerle, C., Bennet, M. \& The MAP Research Team (Boyle, M., MacMillan, HL., Trocmé, N., Goodman, D., Leslie, B., and Moody, B.) (2011). Cannabis Use Among Aboriginal Youth in the Non-Aboriginal Child Protection Services System. First Peoples Child and Family Review, 6(1), 114-125.

Wekerle, C., Leung, E., MacMillan, H.L., Boyle, M., Trocmé, N., \& Waechter, R. (2009). The impact of childhood emotional maltreatment on teen dating violence. Journal of Child Abuse \& Neglect, 33, 45-58.

Wekerle, C., Waechter, R., Leung, E., \& Chen, M. (2009). Chapter 6: Children and youth served by Ontario's Children's Aid Societies. In Profile of Ontario's Children and Youth, Ontario Ministry of Children \& Youth Services Internal Policy Document

Wensing, M., Bosch, M., Grol, R. (2009). Selecting, tailoring, and implementing knowledge translation interventions. In S. Straus, J. Tetroe, and I. Graham (Eds.) Knowledge Translation in Health Care: Moving from Evidence to Practice (pp.94-113). Wiley: UK 